\title{
Genetic component of identification, intensity and pleasantness of odours: a Finnish family study
}

\author{
Antti Knaapila ${ }^{1,2}$, Kaisu Keskitalo ${ }^{1,2}$, Mikko Kallela ${ }^{3}$, Maija Wessman ${ }^{4,5}$, Sampo \\ Sammalisto $^{2}$, Tero Hiekkalinna ${ }^{2,5}$, Aarno Palotie ${ }^{5,6}$, Leena Peltonen ${ }^{2,7}$, Hely Tuorila*,1 and \\ Markus Perola ${ }^{2,7}$ \\ ${ }^{1}$ Department of Food Technology, University of Helsinki, Helsinki, Finland; ${ }^{2}$ Department of Molecular Medicine, \\ National Public Health Institute, Helsinki, Finland; ${ }^{3}$ Department of Neurology, Helsinki University Central Hospital, \\ Helsinki, Finland; ${ }^{4}$ Folkhälsan Research Center, Helsinki, Finland; ${ }^{5}$ Finnish Genome Center, University of Helsinki, \\ Helsinki, Finland; ${ }^{6}$ Department of Clinical Chemistry, University of Helsinki, Helsinki, Finland; ${ }^{7}$ Department of \\ Medical Genetics, University of Helsinki, Helsinki, Finland
}

Although potential odorant receptor genes have been identified, the precise genetic component of perception of odours is still obscure. Although there is some evidence for heritability of a few olfactoryrelated traits, no genome-wide search for loci harboring underlying genes has been published to date. We performed a genome-wide scan to identify loci affecting the identification, intensity and pleasantness of 12 odours (cinnamon, turpentine, lemon, smoke, chocolate, rose, paint thinner, banana, pineapple, gasoline, soap, onion) using 146 Finnish adults from 26 families. Several of these traits showed heritable variation in the families. Suggestive evidence of linkage was found for the pleasantness of cinnamon odour $\left(h^{2}=61 \%\right.$ ) on chromosome $4 q 32.3$ (multipoint logarithm of the odds (LOD) score 3.01), as well as for the perceived intensity of paint thinner odour $\left(h^{2}=31 \%\right)$ on chromosome 2p14 (multipoint LOD score 2.55). As these loci do not contain any known human odorant receptor genes, they may rather harbor genes that affect the central processing than the peripheral detection of the odour signal. Thus, perception of odours is potentially modified by genes other than those encoding odorant receptors.

European Journal of Human Genetics (2007) 15, 596-602. doi:10.1038/sj.ejhg.5201804; published online 7 March 2007

Keywords: olfaction; perception; psychophysics; quantitative trait loci; genetic linkage

\section{Introduction}

The sense of smell is crucial to many animals and important to humans, for example, in finding food and determining its acceptability and palatability, thus having an influence on food choice, nutrition and eventually on health. However, the biology of olfaction was long the least characterized of all senses. ${ }^{1}$ Only in the early 1990s, with the identification of a multigene family

${ }^{*}$ Correspondence: H Tuorila, Department of Food Technology, University of Helsinki, PO Box 66, Fl-00014, University of Helsinki, Finland.

Tel: + 3589191 58216; Fax: + 3589191 58460;

E-mail: hely.tuorila@helsinki.fi

Received 9 August 2006; revised 23 January 2007; accepted 31 January 2007; published online 7 March 2007 encoding odorant receptors (ORs), significant progress has been made in this field. ${ }^{2}$ In humans, genes for Class I (fishlike) ORs were identified clustered on chromosome 11, and Class II (tetrapod-specific) ORs, in contrast, located as clusters on all chromosomes except chromosomes 20 and $\mathrm{Y}^{3}$ OR genes constitute the largest gene family in the human genome. ${ }^{4}$ By systematic searches of the human genome database, two recent studies have identified $339^{5}$ and $388^{6}$ potentially functional OR genes and $297^{5}$ and $414^{6}$ pseudogenes. Variations among individuals and populations in the repertoire of intact and pseudogenic forms of the candidate OR genes was found, and genotypic disparity was suggested to underlie the observed human phenotypic variation. $^{7}$ 
Several studies have been conducted to identify the ligand-specificity of ORs in mouse, rat and human, utilizing cell models (for a review see Mombaerts ${ }^{8}$ ), but genetic studies utilizing psychophysical smell testing of human subjects are rare. Some evidence for genetic control of various olfactory-related traits has been provided by twin studies, ${ }^{9-12}$ and by a study based on pedigree analysis. ${ }^{13}$ However, genome-wide scans for loci influencing olfactory-related traits have not been published so far. Although the potential candidate genes for ORs have been identified, their contribution to the perception of individual odours has not been determined. In addition to the odorant receptor genes, other genes are also likely to modify the perception of odours, as olfactory perception depends not only on peripheral detection but also on central processing. ${ }^{1}$ Psychophysical testing of study subjects combined with genotyping and linkage analysis provides a 'hypothesis-free' approach to the genetics of human olfaction and potentially gives new information on the number and character of the genes and metabolic pathways involved.

We used psychophysical measurement of olfactory-related traits, performed genome-wide marker analyses and adapted statistical analyses of quantitative genetics to examine the genetic background of olfaction. Here, we present the results of a genome-wide screen for loci affecting the olfactoryrelated traits in a study sample emerging from the genetically homogenous population of Finland.

\section{Subjects and methods Subjects}

A total of 146 adult Finns (100 female subjects, 46 male subjects, aged $18-78$ years, mean $49.1 \pm 14.8$ years) from 26 families of the Finnish migraine family study ${ }^{14}$ participated also in this study on a revisit to the migraine study. The study population consisted mainly of subjects with migraine (with or without aura) confirmed by medical diagnosis (84\%) and their healthy relatives (16\%). For analyses the subjects were classified into two groups with respect to smoking: those who had never smoked (58\%) were regarded as non-smokers and those who were smoking or had smoked (42\%) were regarded as smokers. The subjects were asked for a written informed consent and the study plan was approved by the Ethical Committee of the Helsinki University Central Hospital.

\section{Smell testing}

The smell test was carried out at the clinic. Owing to blood sampling, the psychophysical testing was conducted after an overnight fast. Instructions for the smell test were given to the subjects orally and a test administrator was available for questions throughout the test. A questionnaire for background information was mailed to subjects in advance and they returned the filled questionnaire during their visit to the clinic. The questionnaire contained questions for demographic information and smoking habits, together with other issues not reported here.

The brief smell identification test ${ }^{\mathrm{TM}}$ (B-SIT, also known as The cross-cultural smell identification test ${ }^{\mathrm{TM}}, \mathrm{CC}^{\mathrm{S}} \mathrm{SIT}^{15}$ ) was used for smell testing. The B-SIT consists of 12 odours: cinnamon, turpentine, lemon, smoke, chocolate, rose, paint thinner, banana, pineapple, gasoline, soap, and onion. Each odour is microencapsulated in a separate label and released by scratching the label with a tip of a pencil. In its original form, the B-SIT consists of multiple-choice identification tasks with four alternatives given for each odour (forcedchoice). For this study, evaluations of intensity and pleasantness of each odour also were included. Thus, the subjects were asked to scratch and sniff the labels in the order they occur in the test booklet (order was the same for every subject) and then to identify the odour and rate its pleasantness and intensity, in this order. For the evaluations of pleasantness and intensity, the subjects chose an appropriate alternative from 5-point category scales. Categories in the pleasantness scale were labelled as 'very unpleasant' (1), 'fairly unpleasant' (2), 'neither pleasant nor unpleasant' (3), 'fairly pleasant' (4) and 'very pleasant' (5), and in the intensity scale as 'no odour' (1), 'weak odour' (2), 'moderate odour' (3), 'fairly strong odour' (4) and 'very strong odour' (5). Group differences in olfactory performance were explored using analysis of variance, $t$-test and Mann-Whitney $U$-test where appropriate.

\section{Linkage analysis}

Genome-wide marker data (350 polymorphic microsatellite markers) acquired as described previously ${ }^{14}$ were used in linkage analysis. The program Merlin 1.0.1 ${ }^{16}$ was used for variance components linkage analysis and heritability estimation. Before linkage analyses, program PedCheck $1.1^{17}$ was used to check the pedigrees for Mendelian errors, and program PedWipe included in Merlin was used to wipe unlikely genotypes detected by the error-option of Merlin from the pedigree files. Statistical signicance of the heritability estimates were assessed using program QTDT. ${ }^{18}$ Most of the genome-wide analyses were automated using program AUTOGSCAN. ${ }^{19}$

Genetic analyses for intensity and pleasantness evaluations of odours were performed using standardized data in order to avoid bias owing to different use of rating scales by different subjects. Standardizations were made for intensity evaluations as follows (and accordingly for pleasantness evaluations):

$\begin{aligned} & \text { intensity evaluation of } \\ & \text { odour } X \text { by subject } Y\end{aligned}-\begin{aligned} & \text { mean of all intensity } \\ & \text { evaluations by subject } Y\end{aligned}$
standard deviation of all intensity evaluations by subject $Y$

Calculated in this way, the standardized value (z-score) corresponds to the distance of the original value from the mean in SD. 


\section{Results}

The results were based on linkage analysis in a family design in which 146 genome-scanned subjects from 26 families evaluated identity, intensity and pleasantness of 12 odours. Sensory data were combined with the genomewide marker data to locate underlying genetic elements and heritability estimates were determined jointly. Before genetic analysis, effects of age, sex, smoking habits and migraine status were explored.

On average, $10.2 \pm 1.3$ odours of 12 were identified correctly. No linear correlation between the score of correct identifications and age was found, but there was inverse $U$-shaped relationship as the middle-aged (40-59 years) scored best $(\mathrm{F}(2,145)=5.7, P<0.01)$. Instead, slight negative linear correlation was found between age and average intensity evaluation $(r=-0.26, P<0.01)$. Female subjects identified more odours (10.4) than male subjects (9.8; $t=-2.8, P<0.01)$. Female subjects also evaluated odours in general as more intense than male subjects $(t=-3.6$, $P<0.001)$. Of individual odours, female subjects evaluated intensity of chocolate, rose, paint thinner, pineapple, gasoline and soap as higher than male subjects $(Z=-4.6 \ldots-2.3, P<0.05)$, whereas for the other odours no differences were found. No difference in overall pleasantness of odours was found between the genders, but among individual odours, rose odour was evaluated as more pleasant by female subjects $(Z=-3.9, P<0.001)$ and turpentine, paint thinner and gasoline odours by male subjects $(Z=-4.2 \ldots-2.2, P<0.05)$. The smokers (current or past smokers, $n=61$ ) and the non-smokers (never smoked, $n=85$ ) did not significantly differ in their ability to identify odours. Neither were significant differences in pleasantness or intensity evaluations for any odour between these groups found, except for chocolate, which was evaluated as more pleasant by the non-smokers than by the smokers $(Z=-2.4, P<0.05)$. Likewise, no difference in identification scores or in the pleasantness or the intensity evaluations was found between the migraine patients $(n=123)$ and the healthy subjects $(n=23)$, except in the case of gasoline, which was evaluated as more intense by the migraine patients $(Z=-2.5, P<0.05)$.

Half of the odours were identified correctly by $95 \%$ or more of the subjects (Table 1). Among odours that were identified by $<80 \%$ of the subjects (cinnamon, turpentine, lemon, rose and banana), those who identified an odour correctly differed frequently in their intensity and pleasantness evaluations from those who misidentified the odour. Cinnamon and rose odours were evaluated as more intense by those who correctly identified them than those who did not $(Z=-4.2 \ldots-2.2, P<0.05)$. Cinnamon, lemon, rose and banana odours were evaluated as more pleasant $(Z=-6.2 \ldots-2.3, P<0.05)$ and turpentine as less pleasant $(Z=-3.7, P<0.001)$ by the subjects who correctly identified the corresponding odour than the subjects who did not.

\section{Genetic analyses}

Identification The score for correct identification of odours had low heritability $\left(h^{2}=11,13\right.$ and $10 \%$ when analysed without covariates, sex as covariate and sex and age as covariates, respectively) and no evidence of linkage for the trait was found. When individual odours were analysed, only identification of cinnamon, turpentine, lemon, rose and banana were included in linkage analysis in order to avoid false positive results caused by small variation in identification of the other odours (Table 1).

Table 1 Proportion of correct identifications of odours, as well as means and heritabilities for their intensity and pleasant evaluations

\begin{tabular}{|c|c|c|c|c|c|}
\hline \multirow[b]{2}{*}{ Odour $r^{\mathrm{a}}$} & \multirow[b]{2}{*}{ Proportion of correct identification (\%) } & \multicolumn{2}{|c|}{ Intensity } & \multicolumn{2}{|c|}{ Pleasantness } \\
\hline & & Mean (SD) & Heritability $(\%)$ & Mean (SD) & Heritability (\%) \\
\hline 1. Cinnamon ${ }^{b}$ & 73.1 & $2.4(0.6)$ & $0 / 0$ & $3.8(0.7)$ & $62^{\star \star \star} / 65^{\star \star *}$ \\
\hline 2. Turpentine & 54.8 & $2.9(0.7)$ & $0 / 0$ & $2.8(0.8)$ & $32 / 34$ \\
\hline 3. Lemon & 57.5 & $2.7(0.8)$ & $9 / 10$ & $3.2(1.0)$ & $40 * / 42^{*}$ \\
\hline 4. Smoke & 99.3 & $3.1(0.8)$ & $3 / 4$ & $3.2(1.0)$ & $40 * \star / 40 * *$ \\
\hline 5. Chocolate & 95.2 & $3.5(0.8)$ & $31 * / 34 * *$ & $3.9(0.8)$ & $6 / 6$ \\
\hline 6. Rose & 76.7 & $3.2(0.9)$ & $33 * / 34 * *$ & $3.7(0.9)$ & $24 / 17$ \\
\hline 7. Paint thinner & 98.6 & $3.7(0.9)$ & $31 * / 24$ & $2.0(0.9)$ & $0 / 0$ \\
\hline 8. Banana & 74.7 & $3.1(0.8)$ & $28 / 27$ & $3.5(0.9)$ & $0 / 0$ \\
\hline 9. Pineapple & 90.4 & $2.9(0.9)$ & $12 / 12$ & $3.6(0.8)$ & $0 / 0$ \\
\hline 10. Gasoline & 99.3 & $3.7(0.9)$ & $28 * / 24$ & $1.9(0.9)$ & $2 / 2$ \\
\hline 11. Soap & 99.3 & $3.2(0.8)$ & $12 / 12$ & $3.4(0.8)$ & $17 / 15$ \\
\hline 12. Onion & 98.6 & $3.5(0.9)$ & $9 / 7$ & $2.6(0.9)$ & $20 / 21$ \\
\hline Mean & 84.9 & $3.2(0.5)$ & & $3.1(0.4)$ & \\
\hline
\end{tabular}

SD, standard deviation.

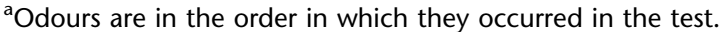

${ }^{b}$ Data for this odour were available from 104 subjects.

${ }^{\mathrm{c} A n a l y s i s ~ w i t h o u t ~ c o v a r i a t e s / a n a l y s i s ~ w i t h ~ s e x ~ a s ~ c o v a r i a t e ; ~ s i g n i f i c a n c e: ~}{ }^{\star} P<0.05,{ }^{\star *} P<0.01,{ }^{\star \star *} P<0.001$. 
Linkage for identification of the five aforementioned analysed odours was not found.

Intensity Perceived intensity of chocolate, rose and paint thinner showed the highest heritabilities $\left(h^{2}=34,34\right.$, and $31 \%$, respectively). For all other odours, heritabilities of the intensity evaluations were below 30\% (Table 1). There was evidence of a suggestive linkage between intensity of paint thinner and marker CHLC.GATA8F03.505 on chromosome 2p14 (multipoint LOD score 2.55; Table 2 and Figures 1 and 2 ). No other evidence of linkage with LOD score $>2.0$ was found.

Pleasantness Pleasantness of cinnamon showed the highest heritability $\left(h^{2}=65 \%\right)$, followed by pleasantness of lemon $\left(h^{2}=42 \%\right)$ and smoke $\left(h^{2}=40 \%\right)$. Heritabilities of other pleasantness evaluations were below $40 \%$ (Table 1). There was evidence of suggestive linkage between pleasantness of cinnamon odour and marker AFM295YE5 on chromosome 4q32.3 (multipoint LOD score 3.01) as well as two other suggestive linkages (Table 2 and Figures 3 and 4). When appropriate covariates were included in linkage analysis, the highest LOD score increased from 2.65 (no covariates) to 2.81 (sex as covariate) and eventually to 3.01 (sex and identification as covariates). No LOD scores over two were found for the pleasantness evaluations of any other odour.

\section{Discussion}

When calculating heritability estimates and LOD scores of continuous traits, it is essential that traits are measured uniformly and thus are comparable between individuals. In psychophysical tests, such as the smell test in the present study, this is not necessarily the case, because all data are self-reported, and individuals can be expected to use rating scales in differing ways. ${ }^{20}$ However, in genetic analyses the relations of measurements among individuals are crucial. Assuming that each individual uses the scales systematically, the differences between individuals in using the scales differently can be corrected by standardizing the absolute values given by each individual. In this way, interindividually comparable data are achieved, as values were expressed relative to the mean. Another advantage resulting from standardization is more continuous-like distribution of a standardized data compared with raw categorical data.

To our knowledge, this is the first study in which the genetic component of olfactory-related traits, namely identification, intensity and pleasantness of odours, was studied by genetic linkage analysis utilising phenotypic data gathered using psychophysical testing of humans. Effects of gender, smoking habits and migraine status on olfactory-related traits were studied to control their confounding effects in the genetic analyses when appropriate. In contrast to smoking habits and migraine status, gender

Table 2 Evidence of linkage to olfactory-related traits

\begin{tabular}{llclrr}
\hline Trait & Covariates & Multipoint LOD score & Marker & Chromosome & Position (cM) \\
\hline Pleasantness of cinnamon odour & sex, identification & 3.01 & AFM295YE5 & $4 \mathrm{q} 32.3$ & 163.65 \\
& & 2.56 & AFM158YE9 & 62.2 & $1 \mathrm{p} 2.93$ \\
& & 2.10 & AFM165XC9 & 1931.3 & 194.98 \\
Intensity of paint thinner odour & $-{ }^{a}$ & 2.55 & CHLC.GATA8F03.505 & $2 \mathrm{p} 14$ & 91.23 \\
\hline
\end{tabular}

cM, centimorgan LOD, logarithm of odds.

ancluding sex as covariate resulted LOD score 1.50.

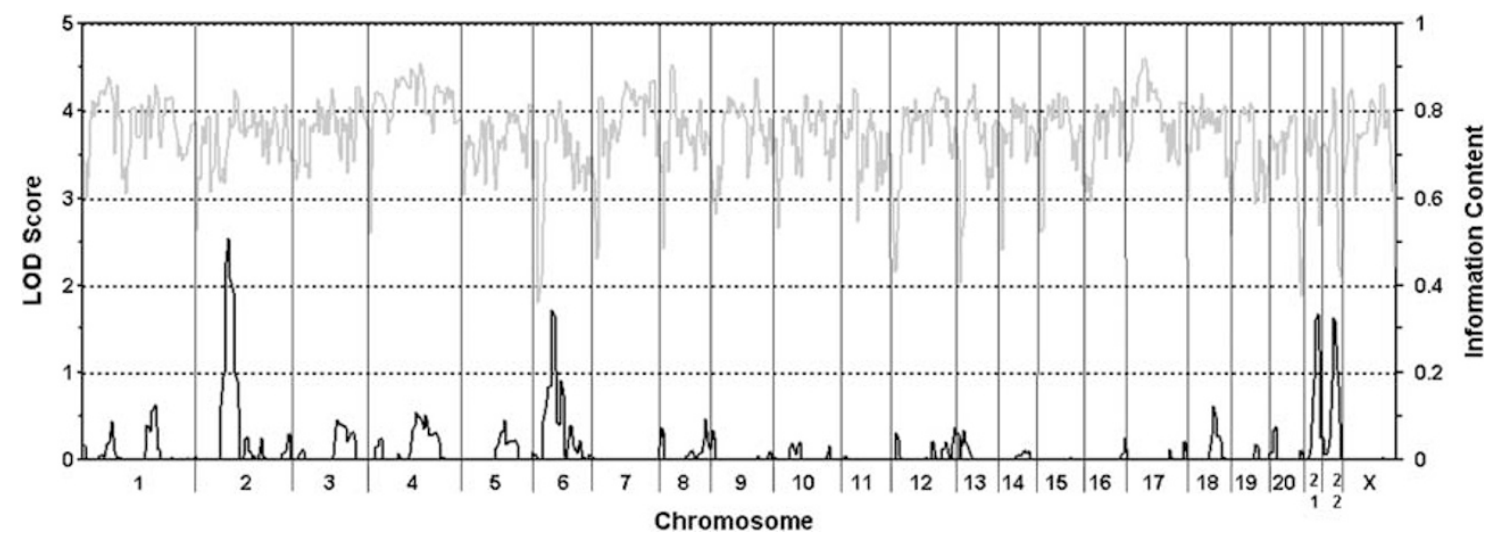

Figure 1 Genome-wide scan for intensity of paint thinner odour (no covariates). Multipoint LOD scores (black) and information content (grey). 
significantly influenced several traits, and therefore the variance components linkage analyses were performed also using sex as covariate. The observed sex differences, especially superiority of female subjects to male subjects in identification of odours, are in line with numerous former studies (for a review see Brand and Millot ${ }^{21}$ ), thus supporting the view that our method of phenotype determination was appropriate and applicable for genetic research as well. In scratch-and-sniff method, individual variation in vigour of scratching may cause variation in the amount of released odorants. This may have increased error variation in intensity evaluations. However, for olfactory stimuli perceived magnitude increases relatively slowly as stimulus magnitude increases. ${ }^{22}$ Thus, the increase in the magnitude of an olfactory stimulus must be relatively large to result in a perceived difference. Hence, minor differences in scratching would not be critical.

Some, but not all, migraine patients have been reported to suffer from olfactory dysfunctions, like hyperosmia, ${ }^{23}$ osmophobia $^{23,24}$ and olfactory hallucinations ${ }^{25-27}$ during the headache phase of their attacks or preceding it as an aura. However, the migraine patients of our study did not

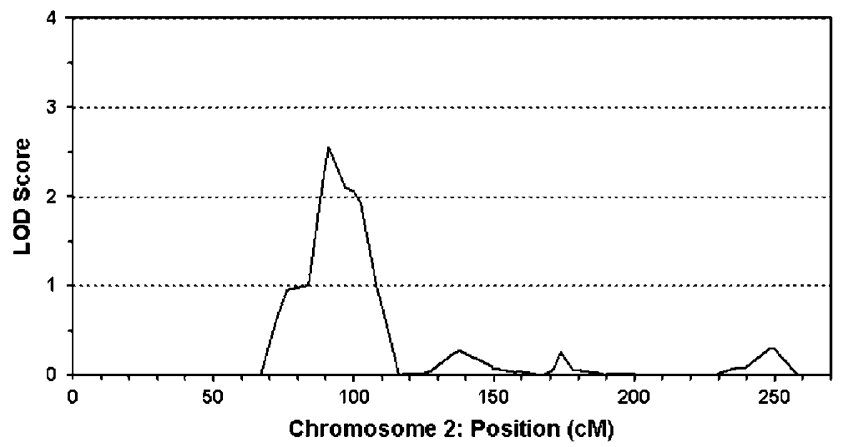

Figure 2 Results of linkage analysis for intensity of paint thinner odour on chromosome 2 in detail (no covariates). report acute migraine during their visit to the clinic and did not suffer from olfactory-related symptoms during the smell test. In addition, there were no significant differences in evaluations of pleasantness and intensity of individual odours between migraine patients and healthy relatives, except for one odour. Thus, we feel that our methodology was applicable to search for general genetic factors underlying variation in pleasantness and intensity of odours despite migraineurs in our study population.

Cognitive inputs seem to influence odour evaluations, whether the information about the odour is given in testing situation or odour itself evokes association. For example, information about negative health effects of an odorant was found to increase intensity ratings of perceived odour and irritation. ${ }^{28}$ Verbal labels of odour stimulus are also known to influence olfactory perception. ${ }^{29}$ Pleasantness and familiarity of odours were found to correlate positively, and earlier experience was suggested to influence perceived intensity. ${ }^{30,31}$ Thus, complex

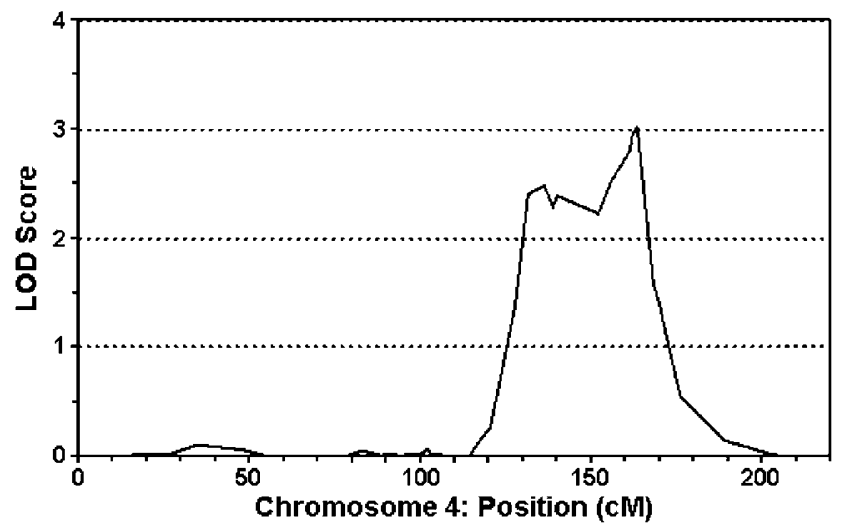

Figure 4 Results of linkage analysis for pleasantness of cinnamon odour on chromosome 4 in detail (sex and identification of the odour as covariates).

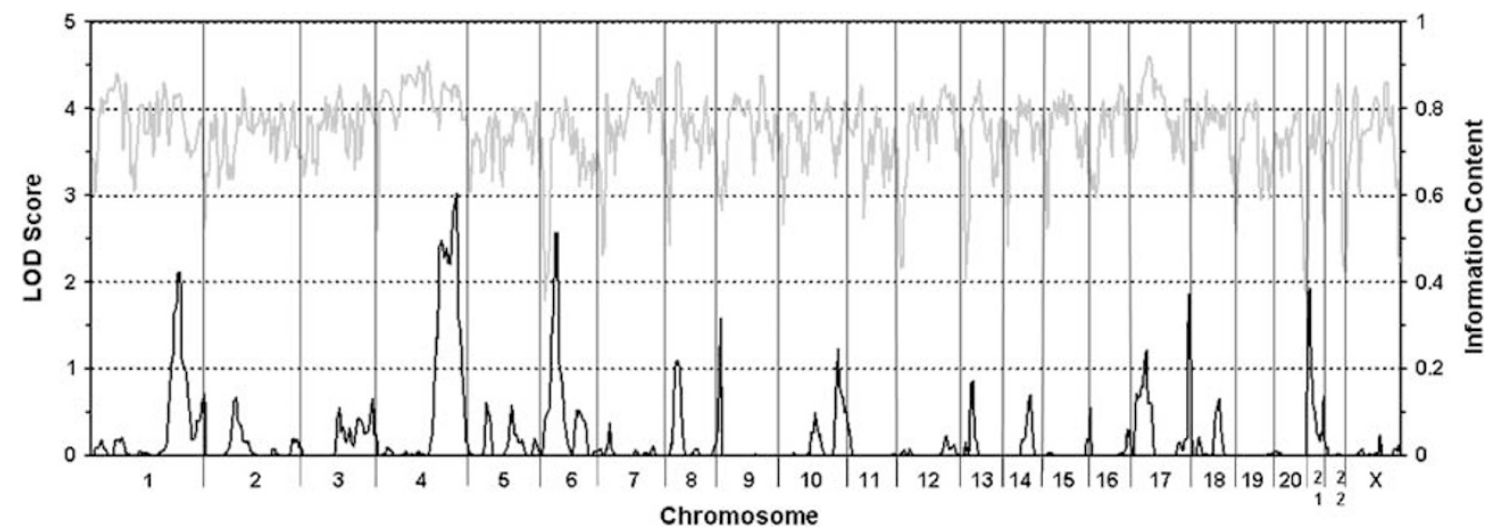

Figure 3 Genome-wide scan for pleasantness of cinnamon odour (sex and identification of the odour as covariates). Multipoint LOD scores (black) and information content (grey). 
environmental influences were probably involved in pleasantness and intensity evaluations also in our data, hindering the detection of potential genetic component. Our finding that an odour tended to be evaluated as more pleasant and more intense when it was identified correctly is consistent with the study of Distel and Hudson. ${ }^{32}$ As the pleasantness evaluation of cinnamon odour differed significantly between those who identified the odour correctly and those who did not, identification of the odour was also applied, together with sex, as covariate for linkage analysis of the trait and consenquently stronger evidence for a linkage was found.

Finkel et $\mathrm{al}^{12}$ studied heritability of mean pleasantness of six odours and reported it to be non-significant, while heritability of pleasantness of individual odours has not been studied earlier. Thus, our finding that pleasantness of cinnamon odour is highly heritable can also be regarded as novel. Likewise, Finkel et $a l^{12}$ studied heritability of the mean intensity of six odours and reported it to be $25 \%$ and only marginally significant $(P<0.10)$, but did not examine the odours separately. However, studies of the perception of odours that were conducted using detection threshold methods have given support for a genetic component of perception of some odours, but not all. Using classical comparison of intraclass correlations between identical and fraternal twins Wysocki and Beauchamp ${ }^{9}$ concluded that the ability to smell androstenone ( $5 \alpha$-androst-16-en-3one) is genetically determined. Using similar methods, Gross-Isseroff et $a l^{10}$ demonstrated a strong genetic component for the perception of isoamyl acetate in addition to androstenone, but did not find genetic effects on the perception of citral and eugenol. In contrast, Segal et $a l^{11}$ failed to show genetic contribution to detection threshold of phenyl ethyl alcohol (PEA), and Hubert et al ${ }^{33}$ of acetic acid, isobutyric acid and cyclohexanone.

In our study, the low heritability we observed for the total score for correct identifications of odours is somewhat in contrast with a study by Finkel et $a l^{12}$ in which significant heritability of $29 \%$ was found for odour identification. However, they used a different smell test (Swedish version of the National Geographic Smell Survey ${ }^{34}$ ) and a twin design. Results from a twin study by Segal et $a l^{11}$ performed using the University of Pennsylvania Smell Identification Test (UPSIT ${ }^{35}$ ), suggested a genetic influence on odour identification for male subjects, but not for female subjects. The finding was based on a significant intraclass correlation for 15 monozygotic male twin pairs, but an estimate for the magnitude of genetic effects was not presented. Low heritability indicating only a small contribution of genetic effects to identification of odours may be explained by the fact that prior experience, learning and familiarity of odours are prominent for evaluation of odour identity. ${ }^{36,37}$

In the present study, detecting the heritability for identification of odours may also be hindered by the low variation (75\% of the subjects scored 10,11 or 12) as well as the non-normal distribution (skewness $=-1.2$, kurtosis $=3.0$ ) of the score for correct identifications. The B-SIT is aimed at rapid screening for olfactory dysfunction, ${ }^{15}$ and was probably not appropriate for detecting sufficient variation among our subjects who in general have the normal sense of smell. Our methods may not have been sensitive enough to detect a genetic component where variation was limited. We did not find any significant or suggestive linkages for odour identification, whether the score for identifications or the individual odours were analysed. This suggests that identification of a single odour is a complicated process influenced by the detection by several ORs as well as signal transduction and central processing of the signal, ${ }^{1}$ thus involving the possible contribution of many genes, of which no single gene is predominant.

The evidence for suggestive linkages to pleasantness of cinnamon and intensity of paint thinner can be considered as novel findings, as no genome-wide searches for loci affecting olfactory-related traits have been performed before the present study. According to Malnic et $a l^{5}$ there are no potentially functional odorant receptor genes on chromosomes 4 or 2 p. Thus, potential genes on chromosome 4 influencing pleasantness of cinnamon odour and on chromosome $2 \mathrm{p}$ influencing perceived intensity of paint thinner odour may have an effect on the central processing of signal rather than on receptors, like Wysocki and Beauchamp ${ }^{9}$ proposed in the case of specific anosmias. They may also influence the regulation of odorant detection by confering variation in odorant-binding proteins ${ }^{38,39}$ or in transcription of odorant receptor genes. The potential genetic elements influencing perception of cinnamon and paint thinner odours probably have broader specificity, because the number of volatile compounds and their combinations with distinctive odours greatly exceeds the number of human genes. Thus, perception of every single odour cannot be influenced by a gene unique to it.

Multipoint LOD scores for the other traits were very low, even for those having heritability over 30\%. Thus, the heritability of these traits may be caused by common family environment rather than by genetic contribution. Alternatively, QTLs (quantitative trait loci) cannot be detected because of the polygenic characteristic of the genetic component, that is, each locus has a very small relative contribution to the observed trait variance. To rule out environmental effects from heritability estimates, modelling of twin data will be a logical next step for further research on heritability. Evidence of linkages, however, supports the genetic component of heritability estimates, and finding the highest heritability and the strongest evidence for linkage for the same trait was hardly a coincidence.

In conclusion, we studied the genetic component of olfactory-related traits in a novel fashion by performing 
genome-wide screens utilising phenotypic data gathered by psychophysical smell testing of humans. For the first time, evidence of suggestive linkage was found for an olfactoryrelated trait, namely pleasantness of cinnamon odour. Phenotypic variation of pleasantness of the odour was shown to have a strong genetic component that may have an influence on regulation of detection or central processing of the odour signal. This suggests that perception of odours may also be modified by genes other than those coding for ORs and warrants further search for underlying genes, as well as studying their specificity and mechanisms of function.

\section{Acknowledgements}

We thank all the subjects for participation in this study. Ms Tanja Moilanen and Ms Kaisu Taskila are highly appreciated for their skilful technical assistance in data collection. Jussi Naukkarinen, BSc, is thanked for helpful comments on language. Support from the Academy of Finland (206 327 (AK, KK), 1210720 (TH), 200923 (AP), 00213 $(M W)$ ), the European Commission (GenomEUtwin project under the 'Quality of Life and Management of the Living Resources' of 5th Framework Programme (no. QLG2-CT-2002-01254), MP, SS), the Finnish Cultural Foundation (TH), the Center of Excellence in Disease Genetics of the Academy of Finland (SS), Biocentrum Helsinki (SS), Helsinki Biomedical Graduate School (SS), Sigrid Juselius Foundation, the Helsinki University Central Hospital, the EuroHead (LSHM-CT2004-504837), the Oxnard Foundation, and the National Institutes of Health (RO1 NS37675 (AP)) are gratefully acknowledged.

\section{References}

1 Keller A, Vosshall LB: Human olfactory psychophysics. Curr Biol 2004; 14: R875-R878.

2 Buck L, Axel R: A novel multigene family may encode odorant receptors: a molecular basis for odor recognition. Cell 1991; 65: $175-187$

3 Glusman G, Yanai I, Rubin I, Lancet D: The complete human olfactory subgenome. Genome Res 2001; 11: 685-702.

4 Olender T, Feldmesser E, Atarot T, Eisenstein M, Lancet D: The olfactory receptor universe - from whole genome analysis to structure and evolution. Genet Mol Res 2004; 3: 545-553.

5 Malnic B, Godfrey PA, Buck LB: The human olfactory receptor gene family. Proc Natl Acad Sci USA 2004; 101: 2584-2589.

6 Niimura Y, Nei M: Evolution of olfactory receptor genes in the human genome. Proc Natl Acad Sci USA 2003; 100: 12235-12240.

7 Menashe I, Man O, Lancet D, Gilad Y: Different noses for different people. Nat Genet 2003; 34: 143-144.

8 Mombaerts P: Genes and ligands for odorant, vomeronasal and taste receptors. Nat Rev Neurosci 2004; 5: 263-278.

9 Wysocki CJ, Beauchamp GK: Ability to smell androstenone is genetically determined. Proc Natl Acad Sci USA 1984; 81: 48994902.

10 Gross-Isseroff R, Ophir D, Bartana A, Voet H, Lancet D: Evidence for genetic determination in human twins of olfactory thresholds for a standard odorant. Neurosci Lett 1992; 141: 115-118.

11 Segal NL, Topolski TD, Wilson SM, Brown KW, Araki L: Twin analysis of odor identification and perception. Physiol Behav 1995; 57: 605-609.

12 Finkel D, Pedersen NL, Larsson M: Olfactory functioning and cognitive abilities: a twin study. J Gerontol Psychol Sci 2001; 56B: P226-P233.

13 Whissell-Buechy D, Amoore JE: Odour-blindness to musk: simple recessive inheritance. Nature 1973; 242: 271-273.
14 Wessman M, Kallela M, Kaunisto MA et al: A susceptibility locus for migraine with aura, on chromosome 4q24. Am J Hum Genet 2002; 70: 652-662.

15 Doty RL, Marcus A, Lee WW: Development of the 12-item crosscultural smell identification test (CC-SIT). Laryngoscope 1996; 106: $353-356$.

16 Abecasis GR, Cherny SS, Cookson WO, Cardon LR: Merlin - rapid analysis of dense genetic maps using sparse gene flow trees. Nat Genet 2002; 30: 97-101.

17 O' Connell JR, Weeks DE: Pedcheck: a program for identification of genotype in linkage analysis. Am J Hum Genet 1998; 63: 259-266.

18 Abecasis GR, Cardon LR, Cookson WOC: A general test of association for quantitative traits in nuclear families. Am J Hum Genet 2000; 66: 279-292.

19 Hiekkalinna T, Terwilliger JD, Sammalisto S, Peltonen L, Perola M: AUTOGSCAN: powerful tools for automated genome-wide linkage and linkage disequilibrium analysis. Twin Res Hum Genet 2005; 8: 16-21.

20 Bartoshuk LM, Duffy VB, Green BG et al: Valid across-group comparisons with labeled scales: the gLMS versus magnitude matching. Physiol Behav 2004; 82: 109-114.

21 Brand G, Millot J-L: Sex differences in human olfaction: between evidence and enigma. Q J Exp Psychol B 2001; 54B: 259-270.

22 Doty RL, Laing DG: Psychophysical measurement of human olfactory function, including odorant mixture assessment; in Doty RL (ed): Handbook of Olfaction and Gustation, 2nd edn, Marcel Dekker, Inc., New York, 2003, pp 203-228.

23 Blau JN, Solomon F: Smell and other sensory disturbances in migraine. J Neurol 1985; 232: 275-276.

24 Kelman L: Osmophobia and taste abnormality in migraineurs: a tertiary care study. Headache 2004; 44: 1019-1023.

25 Wolberg FL, Ziegler DK: Olfactory hallucination in migraine. Arch Neurol 1982; 39: 382

26 Schreiber AO, Calvert PC: Migrainous olfactory hallucinations. Headache 1986; 26: 513-514.

27 Fuller GN, Guiloff RJ: Migrainous olfactory hallucinations. J Neurol Neurosurg Psychiatry 1987; 50: 1688-1690.

28 Dalton P: Cognitive influences on health symptoms from acute chemical exposure. Health Psychol 1999; 18: 579-590.

29 Herz RS: The effect of verbal context on olfactory perception. J Exp Psychol Gen 2003; 132: 595-606.

30 Ayabe-Kanamura S, Schicker I, Laska M et al: Differences in perception of everyday odors: a Japanese-German cross-cultural study. Chem Senses 1998; 23: 31-38.

31 Distel H, Ayabe-Kanamura S, Martinez-Gómez M et al: Perception of everyday odors - correlation between intensity, familiarity, and strength of hedonic judgement. Chem Senses 1999; 24: 191-199.

32 Distel H, Hudson R: Judgement of odor intensity is influenced by subjects' knowledge of the odor source. Chem Senses 2001; 26: $247-251$.

33 Hubert HB, Fabsitz RR, Feinleib M, Brown KS: Olfactory sensitivity in humans: genetic versus environmental control. Science 1980; 208: 607-609.

34 Wysocki CJ, Gilbert AN: National geographic smell survey - effects of age are heterogenous. Ann NY Acad Sci 1989; 561: 12-28.

35 Doty RL, Shaman P, Dann M: Development of the university of Pennsylvania smell identification test: a standardized microencapsulated test of olfactory function. Physiol Behav 1984; 32: 489-502.

36 Stevenson RJ, Boakes RA: A Mnemonic theory of odor perception. Psychol Rev 2003; 110: 340-364.

37 Rabin MD, Cain WS: Odor recognition: familiarity, identifiability, and encoding consistency. J Exp Psychol Learn Mem Cogn 1984; 10: $316-325$.

38 Tegoni M, Pelosi P, Vincent F et al: Mammalian odorant binding proteins. Biochim Biophys Acta 2000; 1482: 229-240.

39 Briand L, Eloit C, Nespoulous C et al: Evidence of an odorantbinding protein in the human olfactory mucus: Location, structural characterization, and odorant-binding properties. Biochemistry 2002; 41: 7241-7252. 